\title{
Modeling of the partial oxidation of glycerol by estimation of its transfer function
}

\author{
Juan Carlos Beltrán-Prieto ${ }^{1, a}$, Karel Kolomazník ${ }^{1}$, Roman Slavík ${ }^{2}$ \\ ${ }^{1}$ Department of Automation and Control Engineering, Faculty of Applied Informatics, Tomas Bata University in Zlín, nám. T. G. Masaryka \\ 5555, 76001 Zlín, Czech Republic \\ ${ }^{2}$ Department of Environmental Engineering Protection, Faculty of Technology, Tomas Bata University in Zlín, nám. T. G. Masaryka 275, \\ 76272 Zlín, Czech Republic
}

\begin{abstract}
The reaction pathway for the conversion of glycerol into different products, namely glyceraldehyde, glyceric acid, glycolic acid, mesoxalic acid and tartronic acid was studied by means of electrochemistry. Multiple Pulse Amperometry technique was used to control the potential during the electrooxidation reaction. The estimation of the transfer function was realized on the basis of dynamic models for the oxidation reaction. The equations obtained in the s-domain were expressed in the time domain using Inverse Laplace transformation to describe the variation of glycerol and products concentration.
\end{abstract}

\section{Introduction}

The partial oxidation of glycerol via heterogeneous redox reaction is a process that is carried out under mild conditions. This allows the minimization of energy waste and by-products generation. This process has been intensively studied by several authors [1-7]. In mentioned works, glyceric acid, $\mathrm{CO}_{2}$, hydroxypyruvate, tartronic acid, mesoxalic acid, glyceraldehyde, or formic acid are among the main products obtained. Although there are reports on glycerol oxidation, there is no record of glyceraldehyde production by electrochemical means on at least pilot plant level.

This makes the research into the production of pure glyceraldehyde a very complicated matter but at the same time more interesting. Most of the publications do not deal in detail with possible mechanism of partial glycerin oxidation and its subsequent quantitative description including experimental verification of the proposed mathematical models.

Determination of transient responses from the models can be performed by using Laplace transform technique; however the solutions obtained are particular to every set of initial conditions. [8] Therefore, the proposal of expressions that can describe the dynamic relation between the input and the output of a process model while being independent of the initial conditions is considered to be valuable. The transfer function is a mathematical representation of a process used in linear time-invariant systems to describe the dynamic properties of the system.

In this study, the electrochemical oxidation of glycerol was analyzed in a three electrode system using platinum as a working electrode.

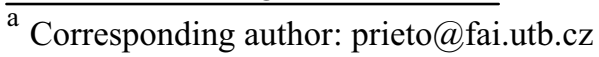

Analysis of reaction products formed during the sustained electrolysis was performed by High Performance Liquid Chromatography (HPLC). Multiple pulse amperometry technique was used to control the potential during the electrooxidation reaction. Proposal of a reaction pathway was performed in order to determine the rate constants, followed by the estimation of the transfer function calculated on the basis of dynamic models for the anodic oxidation.

\section{Materials and methods}

Reagents used for the experiment were purchased from Sigma-Aldrich (Czech Republic), deionized water was used in all procedures (Millipore). Electrochemical oxidation experiments were carried out using an EmStat Potentiostat (made by PalmSens) with computerized control by PSTrace Software to record the data from multiple pulse amperometry measurements.

Analysis of samples for quantification purposes was performed using a Shimadzu HPLC instrument with automatic injection. The system comprised a degassing unit DGU-20A 5R, a pump LC-20AD, an auto sampler SIL-30AC, a column oven CTO-20rA, a Refractive index detector RID-10A, an UV Detector SPD-20A and a communications bus module (control unit) CBM-20A. Data analysis and acquisition was performed with LabSolutions Software.

The HPLC column used was a reversed-phase Aminex HPX-87C (300mm x 7,8mm). Electrochemical experiments were performed as described in [9]. Samples were taken at specific intervals of time and the analysis of 
the glycerol oxidation products was performed using a recent published method [10].

According to the experimental results obtained from electrochemical experiments, a set of differential equations were proposed to test a first-order rate equation considering reversible reactions. A concentration-time curve for the reaction mechanism proposed was obtained by analytical solution of the equations and numerical simulation. The predicted values of the model were compared with the experimental data and the transfer function for the oxidation of glycerol was proposed.

\section{Results}

\subsection{Oxidation at controlled potential by Multiple Pulse Amperometry}

The products formed after the electrochemical reaction were identified and quantified by means of HPLC using two different detectors (Ultraviolet and Refractive index detectors) to ensure the validity of the results. Agreement between both detectors in the quantification of products was always presented.

Figure 1 presents the HPLC chromatogram using Ultraviolet detector of the reaction blend after the partial oxidation of glycerol. Glycerol peak is not present as it does not show absorption at the wavelength applied (210 $\mathrm{nm})$ but it can be easily determined using Refractive index detector. The peak at $8.3 \mathrm{~min}$ is related to the solvent peak.

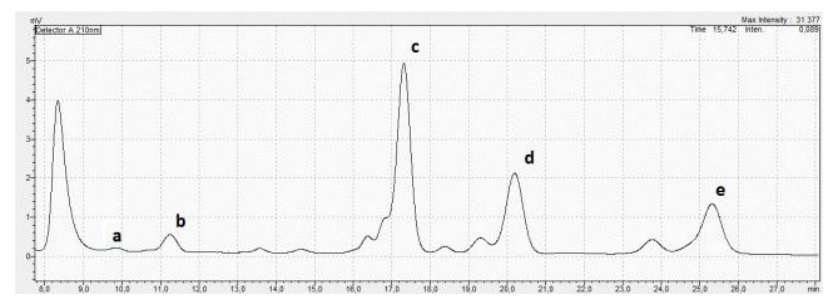

Figure 1. HPLC chromatogram after 5 hours of controlled potential; a) mesoxalic acid, b)tartronic acid, c)glyceraldehyde, d)glyceric acid, e)glycolic acid.

\subsection{Mathematical model and mechanism}

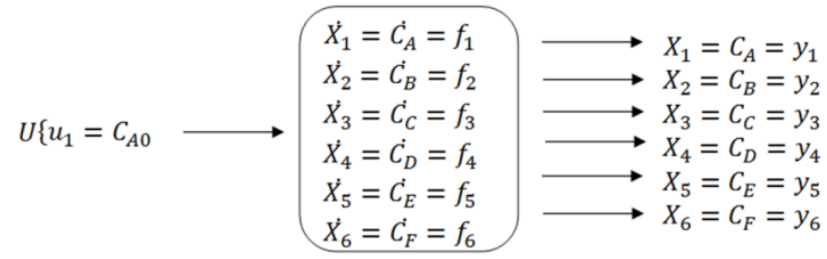

Figure 2. Description of the system including the input, state variable and output.

Figure 2 describes the input-output block diagram of the system. The input is the initial concentration of glycerol. The state and output variables are considered to be the concentration of glycerol $\left(\mathrm{C}_{\mathrm{A}}\right)$, glyceraldehyde $\left(\mathrm{C}_{\mathrm{B}}\right)$, glyceric $\operatorname{acid}\left(\mathrm{C}_{\mathrm{C}}\right)$, tartronic acid $\left(\mathrm{C}_{\mathrm{D}}\right)$, glycolic acid $\left(\mathrm{C}_{\mathrm{E}}\right)$, and mesoxalic acid $\left(\mathrm{C}_{\mathrm{F}}\right)$. The reaction mechanism proposed is presented in Figure 3, considering the oxidation of glycerol into five different products.

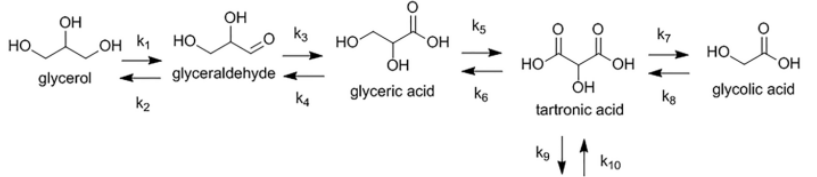

$$
\begin{aligned}
& \text { HO } \prod_{0}^{0}{ }^{\circ}
\end{aligned}
$$

Figure 3. Anodic oxidation pathway proposed considering reversible reactions.

As a result, the mechanism can be described by the set of differential equations presented through (1) to (6)

$$
\begin{gathered}
d C_{A} / d t=-k_{1} C_{A}+k_{2} C_{B} \\
d C_{B} / d t=k_{1} C_{A}-k_{2} C_{B}-k_{3} C_{B}+k_{4} C_{C} \\
d C_{C} / d t=k_{3} C_{B}-k_{4} C_{C}-k_{5} C_{C}+k_{6} C_{D} \\
d C_{D} / d t=k_{5} C_{C}-k_{6} C_{D}-k_{7} C_{D}+k_{8} C_{E}-k_{9} C_{D}+ \\
k_{10} C_{F} \\
d C_{E} / d t=k_{7} C_{D}-k_{8} C_{E} C_{E} \\
d C_{F} / d t=k_{9} C_{D}-k_{10} C_{F} C_{F}
\end{gathered}
$$

The previous equations can be solved numerically to approximate the solutions of the set of ordinary differential equations and to calculate the concentration of glycerol and products formed after evaluation of the respective reaction rate constant. The evaluation of the kinetic constant rates was performed using ERA 3.0 software, which uses the weighted sum of squares as objective function in order to minimize the weighted sum of squares of residual deviations.

Figure 4 present the model fitting to experimental data for concentrations of glycerol and products using the rate constants of $\mathrm{k}_{1}=0.04516 \mathrm{~h}^{-1}, \mathrm{k}_{2}=0.4263 \mathrm{~h}^{-1}, \mathrm{k}_{3}=3.473$ $\mathrm{h}^{-1}, \quad \mathrm{k}_{4}=4.3917 \quad \mathrm{~h}^{-1}, \quad \mathrm{k}_{5}=6.269 \quad \mathrm{~h}^{-1}, \quad \mathrm{k}_{6}=115.176 \quad \mathrm{~h}^{-1}$, $\mathrm{k}_{7}=38.413 \mathrm{~h}^{-1}, \mathrm{k}_{8}=4.345 \mathrm{~h}^{-1}, \mathrm{k}_{9}=3.674 \mathrm{~h}^{-1}, \mathrm{k}_{10}=8.576 \mathrm{~h}^{-1}$. Data is presented in dimensionless values considering the initial concentration of glycerol. Since the anodic oxidation is a heterogeneous process, the rate of the reaction is dependent of the surface concentration of the reactant.

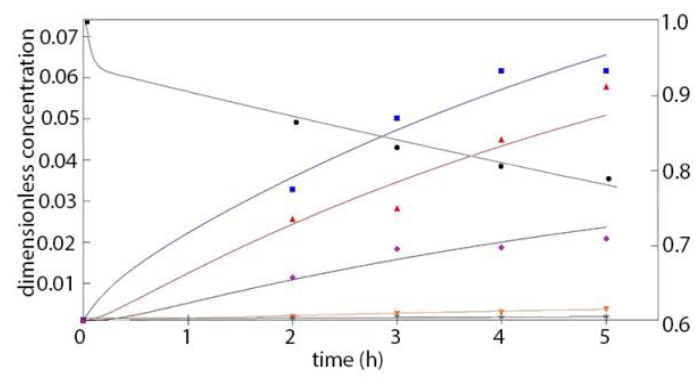

Figure 4 Glycerol and products concentration profile. Comparison between numerical results and experimental data 
for the mechanism proposed Glycerol $(\bullet)$ and products $\left(\backsim C_{B}\right.$; $\Delta C_{C} ; \nabla C_{D} ; C_{E} ;{ }^{*} C_{F}$ ) concentration profile is displayed in the right $\mathrm{y}$-axis and left y-axis respectively.

Equations (1) to (6) represent a vector differential equation as shown in (7) to (15). Considering that the input includes a defined initial concentration of glycerol we obtain the system represented in (10):

$$
\begin{gathered}
\frac{d C}{d \tau}=A \cdot C \\
\tau=0, \quad C=C_{0}
\end{gathered}
$$

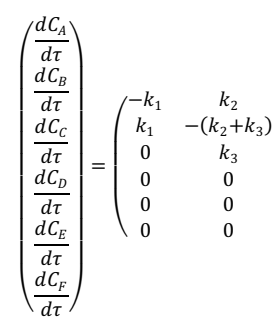

$$
\left.\begin{array}{cccc}
0 & 0 & 0 & 0 \\
k_{4} & 0 & 0 & 0 \\
-\left(k_{4}+k_{5}\right) & k_{6} & 0 & 0 \\
k_{5} & -\left(k_{6}+k_{7}+k_{9}\right) & k_{8} & k_{10} \\
0 & k_{7} & -k_{8} & 0 \\
0 & k_{9} & 0 & -k_{10}
\end{array}\right)\left(\begin{array}{c}
C_{A} \\
C_{B} \\
C_{C} \\
C_{D} \\
C_{E} \\
C_{F}
\end{array}\right)
$$

$$
\left(\begin{array}{c}
C_{A}(0) \\
C_{B}(0) \\
C_{C}(0) \\
C_{D}(0) \\
C_{E}(0) \\
C_{F}(0)
\end{array}\right)=\left(\begin{array}{c}
C_{A 0} \\
0 \\
0 \\
0 \\
0 \\
0
\end{array}\right)
$$

Equations (9) and (10) are represented by the system of equations described in (11)

$$
\begin{gathered}
\Delta \dot{X}_{6 x 1}=A_{6 x 6} \Delta X_{6 x 1}+B_{6 x 1} \Delta U_{1 x 1} \\
\Delta Y=\Delta X
\end{gathered}
$$

Where $A$ is the state matrix of dimension $6 \times 6$ and $B$ is the control matrix of dimension $6 \times 1$. The elements of both matrices A and B correspond to the system presented in (9) and (10) respectively:

By application of the Laplace transform to equation (7) we get:

$$
\begin{gathered}
C_{L}-C_{0}=A \cdot C_{L} \\
C_{L}=(s I-A)^{-1} C_{0}
\end{gathered}
$$

Therefore, in order to determine the transfer function $G(s)$ we need to calculate $(s)=(s I-A)^{-1} B$, as presented next:

$$
(s I-A)=\left(\begin{array}{cccccc}
S & 0 & 0 & 0 & 0 & 0 \\
0 & S & 0 & 0 & 0 & 0 \\
0 & 0 & S & 0 & 0 & 0 \\
0 & 0 & 0 & S & 0 & 0 \\
0 & 0 & 0 & 0 & S & 0 \\
0 & 0 & 0 & 0 & 0 & s
\end{array}\right)-
$$$$
\left(\begin{array}{cccccc}
-k_{1} & k_{2} & 0 & 0 & 0 & 0 \\
k_{1} & -\left(k_{2}+k_{3}\right) & k_{4} & 0 & 0 & 0 \\
0 & k_{3} & -\left(k_{4}+k_{5}\right) & k_{6} & 0 & 0 \\
0 & 0 & k_{5} & -\left(k_{6}+k_{7}+k_{9}\right) & k_{8} & k_{10} \\
0 & 0 & 0 & k_{7} & -k_{8} & 0 \\
0 & 0 & 0 & k_{9} & 0 & -k_{10}
\end{array}\right)
$$

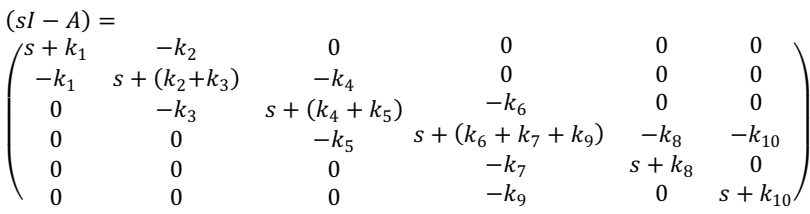

After obtaining the value of $(s I-A)^{-1}$ and multiplying by matrix $\mathrm{B}$, the transfer function of the system can be determined. In this case, numerical values for the transfer function are estimated using the specific values of $C_{A 0}$ (dimensionless value of 1 ) and rate constants $(k)$ calculated previously. As a result, the corresponding transfer functions are given by the following set of equations:

From input $u=C_{A 0}$ to output:

$y_{1}=C_{A} \rightarrow G(s)=$

$\frac{s^{5}+184.744 s^{4}+3653.0028 s^{3}+23865.09 s^{2}+48439.5 s+8034.97}{s^{6}+184.79 s^{5}+3661.33 s^{4}+24026.6 s^{3}+49460.26 s^{2}+9935.52 s-1.7785^{-10}}$

$y_{2}=C_{B} \rightarrow G(s)=$

$0.04516 s^{4}+8.1669 s^{3}+133.813 s^{2}+673.1926 s+851.1834$

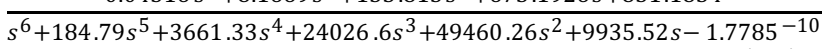

$y_{3}=C_{C} \rightarrow G(s)=$

$0.15684 s^{3}+26.6918 s^{2}+293.4246 s+673.1243$

$s^{6}+184.79 s^{5}+3661.33 s^{4}+24026.6 s^{3}+49460.26 s^{2}+9935.52 s-1.7785^{-10}$

$y_{4}=C_{D} \rightarrow G(s)=$ $0.98323 s^{2}+12.7044 s+36.638$

$s^{6}+184.79 s^{5}+3661.33 s^{4}+24026.6 s^{3}+49460.26 s^{2}+9935.52 s-1.7785^{-10}$

$y_{5}=C_{E} \rightarrow G(s)=$

$37.769 s+323.9067$

$\overline{s^{6}+184.79 s^{5}+3661.33 s^{4}+24026.6 s^{3}+49460.26 s^{2}+9935.52 s-1.7785^{-10}}$

$y_{6}=C_{F} \rightarrow G(s)=$

$\frac{3.6124 s+15.6959}{s^{6}+184.79 s^{5}+3661.33 s^{4}+24026.6 s^{3}+49460.26 s^{2}+9935.52 s-1.7785^{-10}}$

Accordingly, the zero state response can be determined. For this, we firstly require to perform partial fraction expansion of equations (16) to (21) to get:

$$
\begin{aligned}
& Y_{1}(s)=-\frac{0.0003}{s+163.4955}+\frac{0.0003}{s+9.5843}+\frac{0.0003}{s+8.2891}+\frac{0.0002}{s+3.4065}+ \\
& \frac{0.1908}{s+0.2246}+\frac{0.8087}{s} \\
& Y_{2}(s)=\frac{0.0003}{s+163.4955}-\frac{0.003}{s+9.5843}+\frac{0.0012}{s+8.2891}-\frac{0.0039}{s+3.4065}- \\
& \frac{0.0802}{s+0.2246}+\frac{0.0856}{s} \\
& Y_{3}(s)=-\frac{0.0031}{s+9.5843}+\frac{0.0015}{s+8.2891}+\frac{0.0002}{s+3.4065}-\frac{0.0691}{s+0.2246}+ \\
& \frac{0.0677}{s} \\
& Y_{4}(s)=\frac{0.0001}{s+3.4065}-\frac{0.0039}{s+0.2246}+\frac{0.0037}{s}
\end{aligned}
$$




$$
\begin{aligned}
& Y_{5}(s)=-\frac{0.0004}{s+9.5843}-\frac{0.0001}{s+8.2891}+\frac{0.0037}{s+3.4065}-\frac{0.0358}{s+0.2246}+ \\
& \frac{0.0326}{s} \\
& Y_{6}(s)=-\frac{0.0002}{s+9.5843}+\frac{0.0003}{s+8.2891}-\frac{0.0017}{s+0.2246}+\frac{0.0016}{s}
\end{aligned}
$$

Following, the previous equations in the s-domain are then expressed in the time domain using Inverse Laplace transform tables:

$$
\begin{aligned}
& Y_{1}(t)=-0.0003 e^{-163.4955 t}+0.0003 e^{-9.5843 t}+ \\
& 0.0003 e^{-8.2891 t}-0.0002 e^{-3.4065 t}+0.1908 e^{-0.2246 t}+ \\
& 0.8087 \\
& Y_{2}(t)=0.0003 e^{-163.4955 t}-0.003 e^{-9.5843 t}+ \\
& 0.0012 e^{-8.2891 t}-0.0039 e^{-3.4065 t}-0.0802 e^{-0.2246 t}+ \\
& 0.0856 \\
& Y_{3}(t)=-0.0031 e^{-9.5843 t}+0.0015 e^{-8.2891 t}+ \\
& 0.0002 e^{-3.4065 t}-0.0691 e^{-0.2246 t}+0.0677 \\
& Y_{4}(t)=0.0001 e^{-3.4065 t}-0.0039 e^{-0.2246 t}+0.0037
\end{aligned}
$$

$Y_{5}(t)=-0.0004 e^{-9.5843 t}-0.0001 e^{-8.2891 t}+$ $0.0037 e^{-3.4065 t}-0.0358 e^{-0.2246 t}+0.0326$

$Y_{6}(t)=-0.0002 e^{-9.5843 t}+0.0003 e^{-8.2891 t}-$ $0.0017 e^{-0.2246 t}+0.0016$

When analyzing the engineering tasks of process operation and design of control systems, it is important to analyze if the transfer function obtained gives full information of the system. The response of the output/input relation for the variation of glycerol concentration and oxidation products is presented in Figure 5. It is clearly seen a correspondence with the behavior of the experimental and modeling data (Figure 4). As a result, the transfer function obtained describes properly the dynamic relation between the input and output of the oxidation process. Therefore, the partial oxidation of glycerol by electrochemistry demonstrated to be a process that can be modeled by transfer functions.

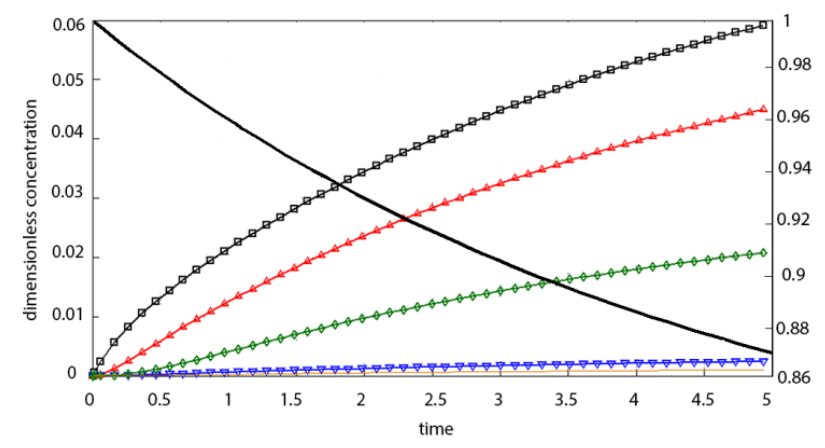

Figure 5. Estimation of the concentration of glycerol (solid dark line) and products formed $\left(\square C_{B} ; \Delta C_{C} ; \nabla C_{D} ; \diamond C_{E} ;-C_{F}\right)$ in the time domain for the anodic oxidation of glycerol experiments. Glycerol concentration is displayed in the right yaxis and products formed in the left y-axis.

\section{Conclusion}

The estimation of the transfer function for the proposed kinetic model of partial oxidation of glycerol was useful in modeling the variation of glycerol derivatives concentration in time. The determination of the system transfer functions and representation of equations in the time domain avoids the analytical solution of the differential equations proposed in the kinetic modeling, which in the case of the full reversible mechanism in every step of the reaction would involve complicated mathematical treatment. Values of rate constants were estimated and agreement between numerical data (from mathematical modeling), experimental data and output using the respective transfer function was observed. As a result, the determined transfer function of the system demonstrated to fully describe the process.

\section{Acknowledgements}

This work was supported by the Ministry of Education, Youth and Sports of the Czech Republic within the National Sustainability Programme project No. LO1303 (MSMT-7778/2014) and also by the European Regional Development Fund under the project CEBIA-Tech No. CZ.1.05/2.1.00/03.0089

\section{References}

1. J. Schnaidt, M. Heinen, D. Denot, Z. Jusys, R. Jürgen Behm, J. Electroanal. Chem. 661, 250-264 (2011).

2. Y. Kwon, S.C.S. Lai, P. Rodriguez, M.T.M. Koper, J. Am. Chem. Soc. 133, 6914-6917 (2011).

3. M. Mougenot, a. Caillard, M. Simoes, S. Baranton, C. Coutanceau, P. Brault, Appl. Catal. B Environ. 107, 372-379 (2011).

4. J. Song, S. Fan, J. Yu, K. Ye, C. Xu, Int. J. Electrochem. Sci. 7, 10842-10850 (2012).

5. A. Serov, U. Martinez, P. Atanassov, Electrochem. Commun. 34, 185-188 (2013).

6. Z. Zhang, L. Xin, W. Li, Appl. Catal. B Environ. 119-120, 40-48 (2012).

7. Z. Zhang, L. Xin, J. Qi, D.J. Chadderdon, W. Li, Appl. Catal. B Environ. 136-137, 29-39 (2013).

8. E.D. Seborg, T.F. Edgar, D. Mellichamp, Process Dynamics and Control, (John Wiley and Sons, Inc. New Jersey, 1989).

9. J.C. Beltran-Prieto, R. Slavik, K. Kolomaznik, Int. J. Electrochem. Sci. 9, 6910-6923 (2014).

10. J.C. Beltrán-Prieto, J. Pecha, V. Kašpárková, K. Kolomazník, J. Liq. Chromatogr. Relat. Technol. 36, 2758-2773 (2013). 